\title{
Superimposed algorithms for the split equilibrium problems and fixed point problems
}

Li-Jun Zhu' ${ }^{1}$ Zhangsong Yao ${ }^{2}$, Yeong-Cheng Liou ${ }^{3,4^{*}}$ and Yonghong Yao

\section{"Correspondence:}

simplex_liou@hotmail.com

${ }^{3}$ Department of Information

Management, Cheng Shiu

University, Kaohsiung, 833, Taiwan

${ }^{4}$ Center for Fundamental Science,

Kaohsiung Medical University,

Kaohsiung, 807, Taiwan

Full list of author information is

available at the end of the article

\begin{abstract}
The split common problems for finding the equilibrium points and fixed points have been studied. A parallel superimposed algorithm is introduced to solve this split common problem. Strong convergence theorems are shown with some analysis techniques.
\end{abstract}

MSC: 49J30; 47H09; 65K10

Keywords: split equilibrium problem; split fixed point; nonexpansive mapping; parallel superimposed algorithms

\section{Introduction}

In the present article, our main purpose is to study the split problem. First, we recall some relevant background in the literature.

\section{Problem 1: the split feasibility problem}

Let $C$ and $Q$ be two nonempty closed convex subsets of Hilbert spaces $H_{1}$ and $H_{2}$, respectively, and let $A: H_{1} \rightarrow H_{2}$ be a bounded linear operator. The problem of finding a point $x^{*}$ such that

$$
x^{*} \in C \text { and } A x^{*} \in Q
$$

is called the split feasibility; it was first introduced by Censor and Elfving [1] in finite dimensional Hilbert spaces. Such problems arise in the field of intensity-modulated radiation therapy when one attempts to describe physical dose constraints and equivalent uniform dose constraints within a single model. When $C \in \mathbb{R}^{N}$ and $Q \in \mathbb{R}^{M}$ are a single pair of sets, Censor and Elfving [1] introduced the simultaneous multi-projections algorithm:

$$
x_{n+1}=A^{-1}\left(\lambda_{1} I+\lambda_{2} A A^{*}\right)^{-1}\left(\lambda_{1} v_{1}^{n+1}+\lambda_{2} A A^{*} v_{2}^{n+1}\right), \quad n \geq 0,
$$

where $\lambda_{1}>0, \lambda_{2}>0, \lambda_{1}+\lambda_{2}=1, v_{i}^{n+1}=P_{C_{i}}^{f_{i}}\left(b_{n}\right)(i=1,2)$, and $b_{n}$ is the solution of the equation

$$
\sum_{i=1}^{2} \lambda_{i} \nabla f_{i}\left(b_{n}\right)=\sum_{i=1}^{2} \nabla f_{i}\left(v_{i}^{n}\right) .
$$

O2014 Zhu et al.; licensee Springer. This is an Open Access article distributed under the terms of the Creative Commons Attribution License (http://creativecommons.org/licenses/by/2.0), which permits unrestricted use, distribution, and reproduction in any medium, provided the original work is properly cited. 
Note that the simultaneous multi-projections algorithm (1.2) involve a matrix inversion $A^{-1}$ at each iterative step. This is very time-consuming, particularly if the dimensions are large. In order to solve this problem, Byrne [2] derived a new algorithm, called the CQalgorithm:

$$
x_{n+1}=P_{C}\left(x_{n}-\tau A^{*}\left(I-P_{Q}\right) A x_{n}\right), \quad n \geq 0,
$$

where $\tau \in\left(0, \frac{2}{L}\right)$ with $L$ being the largest eigenvalue of the matrix $A^{*} A, I$ is the unit matrix or operator, and $P_{C}$ and $P_{Q}$ denote the orthogonal projections onto $C$ and $Q$, respectively. The CQ-algorithm and its variant forms have now been studied for the split feasibility problem; see, for instance [3-13].

\section{Problem 2: the split common fixed point problem}

If every closed convex subset of a Hilbert space is the fixed point set of its associating projection, then the split feasibility problem becomes a special case of the split common fixed point problem of finding a point $x^{*}$ with the property:

$$
x^{*} \in \operatorname{Fix}(U) \text { and } A x^{*} \in \operatorname{Fix}(T) .
$$

This problem was first introduced by Censor and Segal [14] who invented an algorithm which generates a sequence $\left\{x_{n}\right\}$ according to the iterative procedure:

$$
x_{n+1}=U\left(x_{n}-\gamma A^{*}(I-T) A x_{n}\right), \quad n \in \mathbb{N} .
$$

Moudafi [15] extended (1.4) to the following relaxed algorithm:

$$
x_{n+1}=U_{\alpha_{n}}\left(x_{n}+\gamma A^{*}\left(T_{\beta}-I\right) A x_{n}\right), \quad n \in \mathbb{N},
$$

where $\beta \in(0,1), \alpha_{n} \in(0,1)$ are relaxation parameters. Consequently, Wang and $\mathrm{Xu}$ [16] considered a general cyclic algorithm. Very recently, the split problem has also been extended to solve other problems, such as the split monotone variational inclusions and the split variational inequalities, please refer to [15, 17-22] and [23-25].

\section{Problem 3: the equilibrium problem}

Consider the following equilibrium problem: Finding $x^{*} \in C$ such that

$$
F\left(x^{*}, x\right) \geq 0, \quad \forall x \in C,
$$

where $F: C \times C \rightarrow \mathbb{R}$ is a bifunction. We will denote by $\operatorname{EP}(F)$ the set of solutions of (1.5).

The equilibrium problems, in its various forms, found application in optimization problems, fixed point problems, and convex minimization problems; in other words, equilibrium problems are a unified model for problems arising in physics, engineering, economics, and so on (see [26-29]).

Motivated by the split common fixed point problem and the equilibrium problem, $\mathrm{He}$ and $\mathrm{Du}$ [30] presented the following split equilibrium problem and fixed point problem:

Find a point $x^{*} \in \operatorname{Fix}(T) \cap \operatorname{EP}(F)$ such that $A x^{*} \in \operatorname{Fix}(S) \cap \operatorname{EP}(G)$, 
where $\operatorname{Fix}(S)$ and $\operatorname{Fix}(T)$ are the sets of fixed points of two nonlinear mappings $S$ and $T$, respectively, $\operatorname{EP}(F)$ and $\operatorname{EP}(G)$ are the solution sets of two equilibrium problems with bifunctions $F$ and $G$, respectively, and $A$ is a bounded linear mapping. Denote the solution set of (1.6) by

$$
\Gamma=\{x \in \operatorname{Fix}(T) \cap \operatorname{EP}(F): A x \in \operatorname{Fix}(S) \cap \operatorname{EP}(G)\} .
$$

\section{Special cases}

1. If $F=0$ and $G=0$, then (1.6) is reduced to the following split common fixed point problem, which has been considered by many authors, for example, $[14,15,17]$ and [21]:

Find a point $x^{*} \in \operatorname{Fix}(T)$ such that $A x^{*} \in \operatorname{Fix}(S)$.

2. If $S=P_{Q}$ and $T=P_{C}$, then (1.7) is reduced to the split feasibility problem (1.1).

3. If $S$ and $T$ are all identity operators, then (1.6) is reduced to the split equilibrium problem which has been considered in [18]:

Find a point $x^{*} \in \operatorname{EP}(F)$ such that $A x^{*} \in \operatorname{EP}(G)$.

Based on the work in this direction, in this paper we will develop new algorithms to solve the split equilibrium problem and the fixed point problem (1.6). We first introduce a parallel superimposed algorithm. Consequently, strong convergence theorems are shown with some analysis techniques.

\section{Preliminaries}

Let $H$ be a real Hilbert space with inner product $\langle\cdot, \cdot\rangle$ and norm $\|\cdot\|$, respectively. Let $C$ be a nonempty closed convex subset of $H$.

Definition 2.1 A mapping $T: C \rightarrow C$ is called nonexpansive if

$$
\|T x-T y\| \leq\|x-y\|
$$

for all $x, y \in C$.

We will use $\operatorname{Fix}(T)$ to denote the set of fixed points of $T$, that is, $\operatorname{Fix}(T)=\{x \in C: x=T x\}$.

Definition 2.2 A mapping $f: C \rightarrow C$ is called contractive if

$$
\|f(x)-f(y)\| \leq \rho\|x-y\|
$$

for all $x, y \in C$ and for some constant $\rho \in(0,1)$. In this case, we call $f$ is a $\rho$-contraction.

Definition 2.3 A linear bounded operator $B: H \rightarrow H$ is called strongly positive if there exists a constant $\gamma>0$ such that

$$
\langle B x, x\rangle \geq \gamma\|x\|^{2}
$$

for all $x, y \in H$. 
Definition 2.4 We call $P_{C}: H \rightarrow C$ the metric projection if for each $x \in H$

$$
\left\|x-P_{C}(x)\right\|=\inf \{\|x-y\|: y \in C\} .
$$

It is well known that the metric projection $P_{C}: H \rightarrow C$ is characterized by

$$
\left\langle x-P_{C}(x), y-P_{C}(x)\right\rangle \leq 0
$$

for all $x \in H, y \in C$. From this, we can deduce that $P_{C}$ is firmly nonexpansive, that is,

$$
\left\|P_{C}(x)-P_{C}(y)\right\|^{2} \leq\left\langle x-y, P_{C}(x)-P_{C}(y)\right\rangle
$$

for all $x, y \in H$. Hence $P_{C}$ is also nonexpansive.

It is well known that in a real Hilbert space $H$, the following two equalities hold:

$$
\|t x+(1-t) y\|^{2}=t\|x\|^{2}+(1-t)\|y\|^{2}-t(1-t)\|x-y\|^{2}
$$

for all $x, y \in H$ and $t \in[0,1]$, and

$$
\|x+y\|^{2}=\|x\|^{2}+2\langle x, y\rangle+\|y\|^{2}
$$

for all $x, y \in H$. It follows that

$$
\|x+y\|^{2} \leq\|x\|^{2}+2\langle y, x+y\rangle
$$

for all $x, y \in H$.

Throughout this paper, we assume that a bifunction $F: C \times C \rightarrow \mathbb{R}$ satisfies the following conditions:

(H1) $F(x, x)=0$ for all $x \in C$;

(H2) $F$ is monotone, i.e., $F(x, y)+F(y, x) \leq 0$ for all $x, y \in C$;

(H3) for each $x, y, z \in C, \lim _{t \downarrow 0} F(t z+(1-t) x, y) \leq F(x, y)$;

(H4) for each $x \in C, y \mapsto F(x, y)$ is convex and lower semicontinuous.

Lemma 2.5 ([31]) Let $C$ be a nonempty closed convex subset of a real Hilbert space H. Let $F: C \times C \rightarrow \mathbb{R}$ be a bifunction which satisfies conditions (H1)-(H4). Let $r>0$ and $x \in C$. Then there exists $z \in C$ such that

$$
F(z, y)+\frac{1}{r}\langle y-z, z-x\rangle \geq 0, \quad \forall y \in C .
$$

Further, if $U_{r}^{F}(x)=\left\{z \in C: F(z, y)+\frac{1}{r}\langle y-z, z-x\rangle \geq 0, \forall y \in C\right\}$, then the following hold:

(i) $U_{r}^{F}$ is single-valued and $U_{r}^{F}$ is firmly nonexpansive, i.e., for any $x, y \in H$, $\left\|U_{r}^{F} x-U_{r}^{F} y\right\|^{2} \leq\left\langle U_{r}^{F} x-U_{r}^{F} y, x-y\right\rangle$

(ii) $\mathrm{EP}(F)$ is closed and convex and $\mathrm{EP}(F)=\operatorname{Fix}\left(U_{r}^{F}\right)$.

Lemma 2.6 ([32]) Let the mapping $U_{r}^{F}$ be defined as in Lemma 2.5. Then, for $r, s>0$ and $x, y \in H$,

$$
\left\|U_{r}^{F}(x)-U_{s}^{F}(y)\right\| \leq\|x-y\|+\frac{|s-r|}{s}\left\|U_{s}^{F}(y)-y\right\| .
$$


Lemma 2.7 ([33]) Let $\left\{x_{n}\right\}$ and $\left\{y_{n}\right\}$ be two bounded sequences in a Banach space $X$ and let $\left\{\beta_{n}\right\}$ be a sequence in $[0,1]$ with $0<\liminf _{n \rightarrow \infty} \beta_{n} \leq \limsup _{n \rightarrow \infty} \beta_{n}<1$. Suppose that

$$
x_{n+1}=\left(1-\beta_{n}\right) y_{n}+\beta_{n} x_{n}
$$

for all $n \geq 0$ and

$$
\limsup _{n \rightarrow \infty}\left(\left\|y_{n+1}-y_{n}\right\|-\left\|x_{n+1}-x_{n}\right\|\right) \leq 0 .
$$

Then $\lim _{n \rightarrow \infty}\left\|y_{n}-x_{n}\right\|=0$.

Lemma 2.8 ([34]) Let $C$ be a closed convex subset of a real Hilbert space $H$ and let $S: C \rightarrow$ $C$ be a nonexpansive mapping. Then the mapping $I-S$ is demiclosed. That is, if $\left\{x_{n}\right\}$ is a sequence in $C$ such that $x_{n} \rightarrow x^{*}$ weakly and $(I-S) x_{n} \rightarrow y$ strongly, then $(I-S) x^{*}=y$.

Lemma 2.9 ([35]) Assume that $\left\{a_{n}\right\}$ is a sequence of nonnegative real numbers such that

$$
a_{n+1} \leq\left(1-\gamma_{n}\right) a_{n}+\delta_{n}, \quad n \in \mathbb{N}
$$

where $\left\{\gamma_{n}\right\}$ is a sequence in $(0,1)$ and $\left\{\delta_{n}\right\}$ is a sequence such that

(1) $\sum_{n=1}^{\infty} \gamma_{n}=\infty$;

(2) $\lim \sup _{n \rightarrow \infty} \frac{\delta_{n}}{\gamma_{n}} \leq 0$ or $\sum_{n=1}^{\infty}\left|\delta_{n}\right|<\infty$.

Then $\lim _{n \rightarrow \infty} a_{n}=0$.

\section{Main results}

In this section, we introduce our algorithm and prove our main results.

Let $H_{1}$ and $H_{2}$ be two real Hilbert spaces and let $C$ and $D$ be two nonempty closed convex subsets of $H_{1}$ and $H_{2}$, respectively. Let $A: H_{1} \rightarrow H_{2}$ be a bounded linear operator with its adjoint $A^{*}, B$ be a strongly positive bounded linear operator on $H_{1}$ with coefficient $\gamma>0$. Let $f: C \rightarrow C$ be a $\rho$-contraction and $F: C \times C \rightarrow \mathbb{R}$ and $G: D \times D \rightarrow \mathbb{R}$ be two bifunctions satisfying the conditions (H1)-(H4). Let $S: D \rightarrow D$ and $T: C \rightarrow C$ be two nonexpansive mappings.

Algorithm 3.1 Taking $x_{0} \in H_{1}$ arbitrarily, we define a sequence $\left\{x_{n}\right\}$ by the following:

$$
x_{n+1}=\alpha_{n} \sigma f\left(x_{n}\right)+\beta_{n} x_{n}+\left(\left(1-\beta_{n}\right) I-\alpha_{n} B\right) T U_{\lambda_{n}}^{F}\left(x_{n}+\delta A^{*}\left(S U_{\gamma_{n}}^{G}-I\right) A x_{n}\right)
$$

for all $n \in \mathbb{N}$, where $\left\{\lambda_{n}\right\}$ and $\left\{\gamma_{n}\right\}$ are two real number sequences in $(0, \infty), \delta \in\left(0, \frac{1}{\|A\|^{2}}\right)$ and $\sigma>0$ are two constants and $\left\{\alpha_{n}\right\}$ and $\left\{\beta_{n}\right\}$ are two real number sequences in $(0,1)$.

Theorem 3.2 Suppose $\Gamma \neq \emptyset$ and suppose the following conditions hold:

(C1): $\lim _{n \rightarrow \infty} \alpha_{n}=0$ and $\sum_{n=1}^{\infty} \alpha_{n}=\infty$;

(C2): $0<\liminf _{n \rightarrow \infty} \beta_{n} \leq \lim \sup _{n \rightarrow \infty} \beta_{n}<1$;

(C3): $\liminf _{n \rightarrow \infty} \lambda_{n}>0$ and $\lim _{n \rightarrow \infty} \frac{\lambda_{n+1}}{\lambda_{n}}=1$;

(C4): $\liminf _{n \rightarrow \infty} \gamma_{n}>0$ and $\lim _{n \rightarrow \infty} \frac{\gamma_{n+1}}{\gamma_{n}}=1$;

(C5): $\sigma \rho<\gamma$. 
Then the sequence $\left\{x_{n}\right\}$ generated by algorithm (3.1) converges strongly to $p=\operatorname{Proj}_{\Gamma}(\sigma f+$ $I-B) p$, which solves the following VI:

$$
\langle(\sigma f-B) x, y-x\rangle \leq 0, \quad \forall y \in \Gamma .
$$

Proof First, we know that the solution of (3.2) is unique. We denote the unique solution by $p$. That is, $p=\operatorname{Proj}_{\Gamma}(\sigma f+I-B) p$. Then we have $p \in \operatorname{Fix}(T) \cap \operatorname{EP}(F)$ and $A p \in \operatorname{Fix}(S) \cap$ $\operatorname{EP}(G)$. Set $z_{n}=U_{\gamma_{n}}^{G} A x_{n}, y_{n}=x_{n}+\delta A^{*}\left(S U_{\gamma_{n}}^{G}-I\right) A x_{n}$ and $u_{n}=U_{\lambda_{n}}^{F}\left(x_{n}+\delta A^{*}\left(S U_{\gamma_{n}}^{G}-I\right) A x_{n}\right)$ for all $n \in \mathbb{N}$. Then $u_{n}=U_{\lambda_{n}}^{F} y_{n}$. From Lemma 2.5, we know that $U_{\lambda_{n}}^{F}$ and $U_{\gamma_{n}}^{G}$ are firmly nonexpansive. By these facts, we have the following conclusions:

$$
\begin{aligned}
& \left\|z_{n}-A p\right\|=\left\|U_{\gamma_{n}}^{G} A x_{n}-A p\right\| \leq\left\|A x_{n}-A p\right\|, \\
& \left\|u_{n}-p\right\|=\left\|U_{\lambda_{n}}^{F} y_{n}-p\right\| \leq\left\|y_{n}-p\right\|
\end{aligned}
$$

and

$$
\begin{aligned}
\left\|S U_{\gamma_{n}}^{G} A x_{n}-A p\right\|^{2} & \leq\left\|U_{\gamma_{n}}^{G} A x_{n}-A p\right\|^{2} \\
& \leq\left\|A x_{n}-A p\right\|^{2}-\left\|U_{\gamma_{n}}^{G} A x_{n}-A x_{n}\right\|^{2} .
\end{aligned}
$$

Applying Lemma 2.6, we deduce

$$
\begin{aligned}
\left\|u_{n+1}-u_{n}\right\| & =\left\|U_{\lambda_{n+1}}^{F} y_{n+1}-U_{\lambda_{n}}^{F} y_{n}\right\| \\
& \leq\left\|y_{n+1}-y_{n}\right\|+\left|\frac{\lambda_{n+1}-\lambda_{n}}{\lambda_{n+1}}\right|\left\|u_{n+1}-y_{n+1}\right\|
\end{aligned}
$$

and

$$
\begin{aligned}
\left\|z_{n+1}-z_{n}\right\| & =\left\|U_{\gamma_{n+1}}^{G} A x_{n+1}-U_{\gamma_{n}}^{G} A x_{n}\right\| \\
& \leq\left\|A x_{n+1}-A x_{n}\right\|+\left|\frac{\gamma_{n+1}-\gamma_{n}}{\gamma_{n+1}}\right|\left\|z_{n+1}-A x_{n}\right\| .
\end{aligned}
$$

From (3.1), we have

$$
\begin{aligned}
\left\|x_{n+1}-p\right\|= & \left\|\alpha_{n}\left(\sigma f\left(x_{n}\right)-B p\right)+\beta_{n}\left(x_{n}-p\right)+\left(\left(1-\beta_{n}\right) I-\alpha_{n} B\right)\left(T u_{n}-p\right)\right\| \\
\leq & \alpha_{n} \sigma\left\|f\left(x_{n}\right)-f(p)\right\|+\alpha_{n}\|\sigma f(p)-B p\|+\beta_{n}\left\|x_{n}-p\right\| \\
& +\left(1-\beta_{n}-\alpha_{n} \gamma\right)\left\|u_{n}-p\right\| .
\end{aligned}
$$

Using (2.3), we get

$$
\begin{aligned}
\left\|y_{n}-p\right\|^{2}= & \left\|x_{n}-p+\delta A^{*}\left(S z_{n}-A x_{n}\right)\right\|^{2} \\
= & \left\|x_{n}-p\right\|^{2}+\delta^{2}\left\|A^{*}\left(S z_{n}-A x_{n}\right)\right\|^{2} \\
& +2 \delta\left\langle x_{n}-p, A^{*}\left(S z_{n}-A x_{n}\right)\right\rangle .
\end{aligned}
$$


Since $A$ is a linear operator with its adjoint $A^{*}$, we have

$$
\begin{aligned}
\left\langle x_{n}-p, A^{*}\left(S z_{n}-A x_{n}\right)\right\rangle & =\left\langle A\left(x_{n}-p\right), S z_{n}-A x_{n}\right\rangle \\
& =\left\langle A x_{n}-A p+S z_{n}-A x_{n}-\left(S z_{n}-A x_{n}\right), S z_{n}-A x_{n}\right\rangle \\
& =\left\langle S z_{n}-A p, S z_{n}-A x_{n}\right\rangle-\left\|z_{n}-A x_{n}\right\|^{2} .
\end{aligned}
$$

Again from (2.3), we obtain

$$
\left\langle S z_{n}-A p, S z_{n}-A x_{n}\right\rangle=\frac{1}{2}\left(\left\|S z_{n}-A p\right\|^{2}+\left\|S z_{n}-A x_{n}\right\|^{2}-\left\|A x_{n}-A p\right\|^{2}\right) .
$$

From (3.5), (3.10), and (3.11), we have

$$
\begin{aligned}
\left\langle x_{n}-p, A^{*}\left(S z_{n}-A x_{n}\right)\right\rangle= & \frac{1}{2}\left(\left\|S z_{n}-A p\right\|^{2}+\left\|S z_{n}-A x_{n}\right\|^{2}-\left\|A x_{n}-A p\right\|^{2}\right) \\
& -\left\|S z_{n}-A x_{n}\right\|^{2} \\
\leq & \frac{1}{2}\left(\left\|A x_{n}-A p\right\|^{2}-\left\|z_{n}-A x_{n}\right\|^{2}+\left\|S z_{n}-A x_{n}\right\|^{2}\right. \\
& \left.-\left\|A x_{n}-A p\right\|^{2}\right)-\left\|S z_{n}-A x_{n}\right\|^{2} \\
= & -\frac{1}{2}\left\|z_{n}-A x_{n}\right\|^{2}-\frac{1}{2}\left\|S z_{n}-A x_{n}\right\|^{2} .
\end{aligned}
$$

Substituting (3.12) into (3.9) to deduce

$$
\begin{aligned}
\left\|y_{n}-p\right\|^{2} & \leq\left\|x_{n}-p\right\|^{2}+\delta^{2}\|A\|^{2}\left\|S z_{n}-A x_{n}\right\|^{2}+2 \delta\left(-\frac{1}{2}\left\|z_{n}-A x_{n}\right\|^{2}-\frac{1}{2}\left\|S z_{n}-A x_{n}\right\|^{2}\right) \\
& =\left\|x_{n}-p\right\|^{2}+\left(\delta^{2}\|A\|^{2}-\delta\right)\left\|S z_{n}-A x_{n}\right\|^{2}-\delta\left\|z_{n}-A x_{n}\right\|^{2} \\
& \leq\left\|x_{n}-p\right\|^{2} .
\end{aligned}
$$

It follows that

$$
\left\|y_{n}-p\right\| \leq\left\|x_{n}-p\right\|
$$

Thus, from (3.8), we get

$$
\begin{aligned}
\left\|x_{n+1}-p\right\| & \leq \alpha_{n} \sigma \rho\left\|x_{n}-p\right\|+\alpha_{n}\|\sigma f(p)-B p\|+\beta_{n}\left\|x_{n}-p\right\|+\left(1-\beta_{n}-\alpha_{n} \gamma\right)\left\|x_{n}-p\right\| \\
& =\left[1-(\gamma-\sigma \rho) \alpha_{n}\right]\left\|x_{n}-p\right\|+\alpha_{n}\|\sigma f(p)-B p\| \\
& \leq \max \left\{\left\|x_{n}-p\right\|, \frac{\|\sigma f(p)-B p\|}{\gamma-\sigma \rho}\right\} .
\end{aligned}
$$

The boundedness of the sequence $\left\{x_{n}\right\}$ follows.

Next, we estimate $\left\|u_{n+1}-u_{n}\right\|$. Observe that

$$
\begin{aligned}
& \left\|y_{n+1}-y_{n}\right\|^{2} \\
& \quad=\left\|x_{n+1}-x_{n}+\delta\left[A^{*}\left(S z_{n+1}-A x_{n+1}\right)-A^{*}\left(S z_{n}-A x_{n}\right)\right]\right\|^{2}
\end{aligned}
$$




$$
\begin{aligned}
= & \left\|x_{n+1}-x_{n}\right\|^{2}+\delta^{2}\left\|A^{*}\left(S z_{n+1}-A x_{n+1}\right)-A^{*}\left(S z_{n}-A x_{n}\right)\right\|^{2} \\
& +2 \delta\left\langle x_{n+1}-x_{n}, A^{*}\left[\left(S z_{n+1}-A x_{n+1}\right)-\left(S z_{n}-A x_{n}\right)\right]\right\rangle \\
\leq & \left\|x_{n+1}-x_{n}\right\|^{2}+\delta^{2}\|A\|^{2}\left\|S z_{n+1}-S z_{n}-\left(A x_{n+1}-A x_{n}\right)\right\|^{2} \\
& +2 \delta\left\langle A x_{n+1}-A x_{n}, S z_{n+1}-S z_{n}-\left(A x_{n+1}-A x_{n}\right)\right\rangle \\
= & \left\|x_{n+1}-x_{n}\right\|^{2}+\delta^{2}\|A\|^{2}\left\|S z_{n+1}-S z_{n}-\left(A x_{n+1}-A x_{n}\right)\right\|^{2} \\
& +2 \delta\left\langle S z_{n+1}-S z_{n}, S z_{n+1}-S z_{n}-\left(A x_{n+1}-A x_{n}\right)\right\rangle \\
& -2 \delta\left\|S z_{n+1}-S z_{n}-\left(A x_{n+1}-A x_{n}\right)\right\|^{2} \\
= & \left\|x_{n+1}-x_{n}\right\|^{2}+\delta^{2}\|A\|^{2}\left\|S z_{n+1}-S z_{n}-\left(A x_{n+1}-A x_{n}\right)\right\|^{2} \\
& +\delta\left(\left\|S z_{n+1}-S z_{n}\right\|^{2}+\left\|S z_{n+1}-S z_{n}-\left(A x_{n+1}-A x_{n}\right)\right\|^{2}\right. \\
& \left.-\left\|A x_{n+1}-A x_{n}\right\|^{2}\right)-2 \delta\left\|S z_{n+1}-S z_{n}-\left(A x_{n+1}-A x_{n}\right)\right\|^{2} \\
= & \left\|x_{n+1}-x_{n}\right\|^{2}+\left(\delta^{2}\|A\|^{2}-\delta\right)\left\|S z_{n+1}-S z_{n}-\left(A x_{n+1}-A x_{n}\right)\right\|^{2} \\
& +\delta\left(\left\|S z_{n+1}-S z_{n}\right\|^{2}-\left\|A x_{n+1}-A x_{n}\right\|^{2}\right) \\
\leq & \left\|x_{n+1}-x_{n}\right\|^{2}+\left(\delta^{2}\|A\|^{2}-\delta\right)\left\|S z_{n+1}-S z_{n}-\left(A x_{n+1}-A x_{n}\right)\right\|^{2} \\
& +\delta\left(\left\|z_{n+1}-z_{n}\right\|^{2}-\left\|A x_{n+1}-A x_{n}\right\|^{2}\right) .
\end{aligned}
$$

Since $\delta \in\left(0, \frac{1}{\|A\|^{2}}\right)$, we derive by virtue of (3.7) and (3.13) that

$$
\begin{aligned}
\left\|y_{n+1}-y_{n}\right\|^{2} \leq & \left\|x_{n+1}-x_{n}\right\|^{2} \\
& +\delta\left|\frac{\gamma_{n+1}-\gamma_{n}}{\gamma_{n+1}}\right|\left(\left\|z_{n+1}-z_{n}\right\|+\left\|A x_{n+1}-A x_{n}\right\|\right) .
\end{aligned}
$$

According to (3.6) and (3.14), we have

$$
\begin{aligned}
\left\|u_{n+1}-u_{n}\right\|^{2}= & \left\|U_{\lambda_{n+1}}^{F} y_{n+1}-U_{\lambda_{n}}^{F} y_{n}\right\|^{2} \\
\leq & \left(\left\|y_{n+1}-y_{n}\right\|+\left|\frac{\lambda_{n+1}-\lambda_{n}}{\lambda_{n+1}}\right|\left\|u_{n+1}-y_{n+1}\right\|\right)^{2} \\
\leq & \left\|y_{n+1}-y_{n}\right\|^{2}+\left|\frac{\lambda_{n+1}-\lambda_{n}}{\lambda_{n+1}}\right|\left(2\left\|y_{n+1}-y_{n}\right\|\left\|u_{n+1}-y_{n+1}\right\|\right. \\
& \left.+\left|\frac{\lambda_{n+1}-\lambda_{n}}{\lambda_{n+1}}\right|\left\|u_{n+1}-y_{n+1}\right\|^{2}\right) \\
\leq & \left\|x_{n+1}-x_{n}\right\|^{2}+\left|\frac{\lambda_{n+1}-\lambda_{n}}{\lambda_{n+1}}\right|\left(2\left\|y_{n+1}-y_{n}\right\|\left\|u_{n+1}-y_{n+1}\right\|\right. \\
& \left.+\left|\frac{\lambda_{n+1}-\lambda_{n}}{\lambda_{n+1}}\right|\left\|u_{n+1}-y_{n+1}\right\|^{2}\right) \\
& +\delta\left|\frac{\gamma_{n+1}-\gamma_{n}}{\gamma_{n+1}}\right|\left(\left\|z_{n+1}-z_{n}\right\|+\left\|A x_{n+1}-A x_{n}\right\|\right) \\
\leq & \left\|x_{n+1}-x_{n}\right\|^{2}+\left(\left|\frac{\lambda_{n+1}-\lambda_{n}}{\lambda_{n+1}}\right|+\delta\left|\frac{\gamma_{n+1}-\gamma_{n}}{\gamma_{n+1}}\right|\right) M,
\end{aligned}
$$


where $M>0$ is a constant such that

$$
\begin{aligned}
& \sup _{n}\left\{2\left\|y_{n+1}-y_{n}\right\|\left\|u_{n+1}-y_{n+1}\right\|+\left|\frac{\lambda_{n+1}-\lambda_{n}}{\lambda_{n+1}}\right|\left\|u_{n+1}-y_{n+1}\right\|^{2}\right. \\
& \left.\quad+\delta\left(\left\|z_{n+1}-z_{n}\right\|+\left\|A x_{n+1}-A x_{n}\right\|\right)\right\} \leq M .
\end{aligned}
$$

Therefore,

$$
\left\|u_{n+1}-u_{n}\right\| \leq\left\|x_{n+1}-x_{n}\right\|+\sqrt{\left(\left|\frac{\lambda_{n+1}-\lambda_{n}}{\lambda_{n+1}}\right|+\delta\left|\frac{\gamma_{n+1}-\gamma_{n}}{\gamma_{n+1}}\right|\right) M} .
$$

From (3.1), we write $x_{n+1}=\beta_{n} x_{n}+\left(1-\beta_{n}\right) w_{n}$ where $w_{n}=T u_{n}+\frac{\alpha_{n}}{1-\beta_{n}}\left(\sigma f\left(x_{n}\right)-B T u_{n}\right)$ for all $n \in \mathbb{N}$. Then we have

$$
\begin{aligned}
& \left\|w_{n+1}-w_{n}\right\| \\
& =\left\|T u_{n+1}-T u_{n}+\frac{\alpha_{n+1}}{1-\beta_{n+1}}\left(\sigma f\left(x_{n+1}\right)-B T u_{n+1}\right)-\frac{\alpha_{n}}{1-\beta_{n}}\left(\sigma f\left(x_{n}\right)-B T u_{n}\right)\right\| \\
& \leq\left\|T u_{n+1}-T u_{n}\right\|+\frac{\alpha_{n+1}}{1-\beta_{n+1}}\left\|\sigma f\left(x_{n+1}\right)-B T u_{n+1}\right\|+\frac{\alpha_{n}}{1-\beta_{n}}\left\|\sigma f\left(x_{n}\right)-B T u_{n}\right\| \\
& \leq\left\|u_{n+1}-u_{n}\right\|+\frac{\alpha_{n+1}}{1-\beta_{n+1}}\left\|\sigma f\left(x_{n+1}\right)-B T u_{n+1}\right\|+\frac{\alpha_{n}}{1-\beta_{n}}\left\|\sigma f\left(x_{n}\right)-B T u_{n}\right\| \\
& \leq\left\|x_{n+1}-x_{n}\right\|+\sqrt{\left(\left|\frac{\lambda_{n+1}-\lambda_{n}}{\lambda_{n+1}}\right|+\delta\left|\frac{\gamma_{n+1}-\gamma_{n}}{\gamma_{n+1}}\right|\right) M} \\
& \quad+\frac{\alpha_{n+1}}{1-\beta_{n+1}}\left\|\sigma f\left(x_{n+1}\right)-B T u_{n+1}\right\|+\frac{\alpha_{n}}{1-\beta_{n}}\left\|\sigma f\left(x_{n}\right)-B T u_{n}\right\| .
\end{aligned}
$$

Noting the condition (C1) and the boundedness of the sequences $\left\{u_{n+1}\right\},\left\{y_{n+1}\right\},\left\{z_{n+1}\right\}$, $\left\{A x_{n}\right\},\left\{f\left(x_{n}\right)\right\}$, and $\left\{B T u_{n}\right\}$, we have

$$
\limsup _{n \rightarrow \infty}\left(\left\|w_{n+1}-w_{n}\right\|-\left\|x_{n+1}-x_{n}\right\|\right) \leq 0
$$

By Lemma 2.7, we deduce

$$
\lim _{n \rightarrow \infty}\left\|x_{n}-w_{n}\right\|=0
$$

Hence,

$$
\lim _{n \rightarrow \infty}\left\|x_{n+1}-x_{n}\right\|=\lim _{n \rightarrow \infty}\left(1-\beta_{n}\right)\left\|x_{n}-w_{n}\right\|=0 .
$$

Since $x_{n+1}-x_{n}=\alpha_{n}\left(\sigma f\left(x_{n}\right)-B T u_{n}\right)+\left(1-\beta_{n}\right)\left(T u_{n}-x_{n}\right)$, we obtain

$$
\left\|T u_{n}-x_{n}\right\| \leq \frac{1}{\beta_{n}}\left\{\alpha_{n}\left\|\sigma f\left(x_{n}\right)-B T u_{n}\right\|+\left\|x_{n+1}-x_{n}\right\|\right\} .
$$

Thus,

$$
\lim _{n \rightarrow \infty}\left\|x_{n}-T u_{n}\right\|=0 .
$$


Using the firmly nonexpansiveness of $U_{\lambda_{n}}^{F}$, we have

$$
\begin{aligned}
\left\|u_{n}-p\right\|^{2}= & \left\|U_{\lambda_{n}}^{F} y_{n}-p\right\|^{2} \\
\leq & \left\|y_{n}-p\right\|^{2}-\left\|U_{\lambda_{n}}^{F} y_{n}-y_{n}\right\|^{2} \\
= & \left\|y_{n}-p\right\|^{2}-\left\|u_{n}-y_{n}\right\|^{2} \\
= & \left\|y_{n}-p\right\|^{2}-\left\|u_{n}-x_{n}-\delta A^{*}\left(S U_{\gamma_{n}}^{G}-I\right) A x_{n}\right\|^{2} \\
= & \left\|y_{n}-p\right\|^{2}-\left\|u_{n}-x_{n}\right\|^{2}-\delta^{2}\left\|A^{*}\left(S U_{\gamma_{n}}^{G}-I\right) A x_{n}\right\|^{2} \\
& +2 \delta\left\langle u_{n}-x_{n}, A^{*}\left(S U_{\gamma_{n}}^{G}-I\right) A x_{n}\right\rangle .
\end{aligned}
$$

Applying (2.4) to (3.1) to deduce

$$
\begin{aligned}
&\left\|x_{n+1}-p\right\|^{2} \\
&=\left\|\alpha_{n}\left(\sigma f\left(x_{n}\right)-B p\right)+\beta_{n}\left(x_{n}-T u_{n}\right)+\left(I-\alpha_{n} B\right)\left(T u_{n}-p\right)\right\|^{2} \\
& \leq\left\|\left(I-\alpha_{n} B\right)\left(T u_{n}-p\right)+\beta_{n}\left(x_{n}-T u_{n}\right)\right\|^{2}+2 \alpha_{n}\left\langle\sigma f\left(x_{n}\right)-B p, x_{n+1}-p\right\rangle \\
& \leq\left[\left\|I-\alpha_{n} B\right\|\left\|T u_{n}-p\right\|+\beta_{n}\left\|x_{n}-T u_{n}\right\|\right]^{2}+2 \alpha_{n}\left\|\sigma f\left(x_{n}\right)-B p\right\|\left\|x_{n+1}-p\right\| \\
& \leq {\left[\left(1-\alpha_{n} \gamma\right)\left\|u_{n}-p\right\|+\beta_{n}\left\|x_{n}-T u_{n}\right\|\right]^{2}+2 \alpha_{n}\left\|\sigma f\left(x_{n}\right)-B p\right\|\left\|x_{n+1}-p\right\| } \\
&=\left(1-\alpha_{n} \gamma\right)^{2}\left\|u_{n}-p\right\|^{2}+\beta_{n}^{2}\left\|x_{n}-T u_{n}\right\|^{2}+2\left(1-\alpha_{n} \gamma\right) \beta_{n}\left\|u_{n}-p\right\|\left\|x_{n}-T u_{n}\right\| \\
& \quad+2 \alpha_{n}\left\|\sigma f\left(x_{n}\right)-B p\right\|\left\|x_{n+1}-p\right\| .
\end{aligned}
$$

It follows from (3.19) that

$$
\begin{aligned}
\left\|x_{n+1}-p\right\|^{2} \leq & \left\|x_{n}-p\right\|^{2}-\left\|u_{n}-y_{n}\right\|^{2}+\beta_{n}^{2}\left\|x_{n}-T u_{n}\right\|^{2} \\
& +2\left(1-\alpha_{n} \gamma\right) \beta_{n}\left\|u_{n}-p\right\|\left\|x_{n}-T u_{n}\right\| \\
& +2 \alpha_{n}\left\|\sigma f\left(x_{n}\right)-B p\right\|\left\|x_{n+1}-p\right\| .
\end{aligned}
$$

Then

$$
\begin{aligned}
\left\|u_{n}-y_{n}\right\|^{2} \leq & \left\|x_{n}-p\right\|^{2}-\left\|x_{n+1}-p\right\|^{2}+\beta_{n}^{2}\left\|x_{n}-T u_{n}\right\|^{2} \\
& +2\left(1-\alpha_{n} \gamma\right) \beta_{n}\left\|u_{n}-p\right\|\left\|x_{n}-T u_{n}\right\|+2 \alpha_{n}\left\|\sigma f\left(x_{n}\right)-B p\right\|\left\|x_{n+1}-p\right\| \\
\leq & \left(\left\|x_{n}-p\right\|+\left\|x_{n+1}-p\right\|\right)\left\|x_{n+1}-x_{n}\right\|+\beta_{n}^{2}\left\|x_{n}-T u_{n}\right\|^{2} \\
& +2\left(1-\alpha_{n} \gamma\right) \beta_{n}\left\|u_{n}-p\right\|\left\|x_{n}-T u_{n}\right\|+2 \alpha_{n}\left\|\sigma f\left(x_{n}\right)-B p\right\|\left\|x_{n+1}-p\right\| .
\end{aligned}
$$

This together with (C1), (3.17), and (3.18) implies that

$$
\lim _{n \rightarrow \infty}\left\|u_{n}-y_{n}\right\|=0
$$

From (3.20), we have

$$
\begin{aligned}
\left\|x_{n+1}-p\right\|^{2} \leq & \left(1-\alpha_{n} \gamma\right)^{2}\left\|u_{n}-p\right\|^{2}+\beta_{n}^{2}\left\|x_{n}-T u_{n}\right\|^{2} \\
& +2\left(1-\alpha_{n} \gamma\right) \beta_{n}\left\|u_{n}-p\right\|\left\|x_{n}-T u_{n}\right\|+2 \alpha_{n}\left\|\sigma f\left(x_{n}\right)-B p\right\|\left\|x_{n+1}-p\right\|
\end{aligned}
$$




$$
\begin{aligned}
\leq & \left\|y_{n}-p\right\|^{2}+\beta_{n}^{2}\left\|x_{n}-T u_{n}\right\|^{2}+2\left(1-\alpha_{n} \gamma\right) \beta_{n}\left\|u_{n}-p\right\|\left\|x_{n}-T u_{n}\right\| \\
& +2 \alpha_{n}\left\|\sigma f\left(x_{n}\right)-B p\right\|\left\|x_{n+1}-p\right\| \\
\leq & \left\|x_{n}-p\right\|^{2}+\left(\delta^{2}\|A\|^{2}-\delta\right)\left\|S z_{n}-A x_{n}\right\|^{2}-\delta\left\|z_{n}-A x_{n}\right\|^{2} \\
& +\beta_{n}^{2}\left\|x_{n}-T u_{n}\right\|^{2}+2\left(1-\alpha_{n} \gamma\right) \beta_{n}\left\|u_{n}-p\right\|\left\|x_{n}-T u_{n}\right\| \\
& +2 \alpha_{n}\left\|\sigma f\left(x_{n}\right)-B p\right\|\left\|x_{n+1}-p\right\| .
\end{aligned}
$$

Hence,

$$
\begin{aligned}
(\delta- & \left.\delta^{2}\|A\|^{2}\right)\left\|S z_{n}-A x_{n}\right\|^{2}+\delta\left\|z_{n}-A x_{n}\right\|^{2} \\
\leq & \left\|x_{n}-p\right\|^{2}-\left\|x_{n+1}-p\right\|^{2}+\beta_{n}^{2}\left\|x_{n}-T u_{n}\right\|^{2} \\
& +2\left(1-\alpha_{n} \gamma\right) \beta_{n}\left\|u_{n}-p\right\|\left\|x_{n}-T u_{n}\right\|+2 \alpha_{n}\left\|\sigma f\left(x_{n}\right)-B p\right\|\left\|x_{n+1}-p\right\| \\
\leq & \left(\left\|x_{n}-p\right\|+\left\|x_{n+1}-p\right\|\right)\left\|x_{n+1}-x_{n}\right\|+\beta_{n}^{2}\left\|x_{n}-T u_{n}\right\|^{2} \\
& +2\left(1-\alpha_{n} \gamma\right) \beta_{n}\left\|u_{n}-p\right\|\left\|x_{n}-T u_{n}\right\|+2 \alpha_{n}\left\|\sigma f\left(x_{n}\right)-B p\right\|\left\|x_{n+1}-p\right\|,
\end{aligned}
$$

which implies that

$$
\lim _{n \rightarrow \infty}\left\|S z_{n}-A x_{n}\right\|=\lim _{n \rightarrow \infty}\left\|z_{n}-A x_{n}\right\|=0 .
$$

So,

$$
\lim _{n \rightarrow \infty}\left\|S z_{n}-z_{n}\right\|=0
$$

Note that

$$
\begin{aligned}
\left\|y_{n}-x_{n}\right\| & =\left\|\delta A^{*}\left(S U_{\gamma_{n}}^{G}-I\right) A x_{n}\right\| \\
& \leq \delta\|A\|\left\|S z_{n}-A x_{n}\right\| .
\end{aligned}
$$

Therefore,

$$
\lim _{n \rightarrow \infty}\left\|x_{n}-y_{n}\right\|=0
$$

From (3.18), (3.22), and (3.24), we get

$$
\lim _{n \rightarrow \infty}\left\|x_{n}-T x_{n}\right\|=0
$$

Now, we show that $\lim \sup _{n \rightarrow \infty}\left\langle(\sigma f-B) p, x_{n}-p\right\rangle \leq 0$. Choose a subsequence $\left\{x_{n_{i}}\right\}$ of $\left\{x_{n}\right\}$ such that

$$
\limsup _{n \rightarrow \infty}\left\langle(\sigma f-B) p, x_{n}-p\right\rangle=\lim _{i \rightarrow \infty}\left\langle(\sigma f-B) p, x_{n_{i}}-p\right\rangle
$$

Since the sequence $\left\{x_{n_{i}}\right\}$ is bounded, we can choose a subsequence $\left\{x_{n_{i_{j}}}\right\}$ of $\left\{x_{n_{i}}\right\}$ such that $x_{n_{i_{j}}} \rightarrow z$. For the sake of convenience, we assume (without loss of generality) that $x_{n_{i}} \rightarrow z$. 
Consequently, we derive from the above conclusions that

$$
y_{n_{i}} \rightarrow z, \quad u_{n_{i}} \rightarrow z, \quad A x_{n_{i}} \rightarrow z \quad \text { and } \quad z_{n_{i}} \rightarrow A z .
$$

By the demi-closed principle of the nonexpansive mappings $S$ and $T$ (see Lemma 2.8), we deduce $z \in \operatorname{Fix}(T)$ and $A z \in \operatorname{Fix}(S)$ (according to (3.25) and (3.23), respectively).

Next, we show that $z \in \operatorname{EP}(F)$. Since $u_{n}=U_{\lambda_{n}}^{F} y_{n}$, we have

$$
F\left(u_{n}, y\right)+\frac{1}{\lambda_{n}}\left\langle y-u_{n}, u_{n}-y_{n}\right\rangle \geq 0, \quad \forall y \in C .
$$

It follows from the monotonicity of $F$ that

$$
\frac{1}{\lambda_{n}}\left\langle y-u_{n}, u_{n}-y_{n}\right\rangle \geq F\left(y, u_{n}\right),
$$

and hence

$$
\left\langle y-u_{n_{i}}, \frac{u_{n_{i}}-y_{n_{i}}}{\lambda_{n_{i}}}\right\rangle \geq F\left(y, u_{n_{i}}\right) .
$$

Since $\left\|u_{n}-y_{n}\right\| \rightarrow 0, u_{n_{i}} \rightarrow z$, and $\liminf _{n \rightarrow \infty} \lambda_{n}>0$, we obtain $\frac{u_{n_{i}}-y_{n_{i}}}{\lambda_{n_{i}}} \rightarrow 0$. It follows that $0 \geq F(y, z)$. For $t$ with $0<t \leq 1$ and $y \in C$, let $y_{t}=t y+(1-t) z \in C$. It follows that $F\left(y_{t}, z\right) \leq 0$. So,

$$
0=F\left(y_{t}, y_{t}\right) \leq t F\left(y_{t}, y\right)+(1-t) F\left(y_{t}, z\right) \leq t F\left(y_{t}, y\right) .
$$

Therefore, $0 \leq F\left(y_{t}, y\right)$. Thus $0 \leq F(z, y)$. This implies that $z \in \operatorname{EP}(F)$. Similarly, we can prove that $A z \in \operatorname{EP}(G)$. To this end, we deduce $z \in \operatorname{Fix}(T) \cap \operatorname{EP}(F)$ and $A z \in \operatorname{Fix}(S) \cap \operatorname{EP}(G)$. That is to say, $z \in \Gamma$. Therefore,

$$
\begin{aligned}
\limsup _{n \rightarrow \infty}\left\langle(\sigma f-B) p, x_{n}-p\right\rangle & =\lim _{i \rightarrow \infty}\left\langle(\sigma f-B) p, x_{n_{i}}-p\right\rangle \\
& =\lim _{i \rightarrow \infty}\langle(\sigma f-B) p, z-p\rangle \\
& \leq 0 .
\end{aligned}
$$

Finally, we prove $x_{n} \rightarrow p$. From (3.1), we have

$$
\begin{aligned}
&\left\|x_{n+1}-p\right\|^{2} \\
&=\left\langle\alpha_{n}\left(\sigma f\left(x_{n}\right)-B p\right)+\beta_{n}\left(x_{n}-p\right)+\left(\left(1-\beta_{n}\right) I-\alpha_{n} B\right)\left(T u_{n}-p\right), x_{n+1}-p\right\rangle \\
&=\alpha_{n}\left|\sigma f\left(x_{n}\right)-B p, x_{n+1}-p\right\rangle+\beta_{n}\left\langle x_{n}-p, x_{n+1}-p\right\rangle \\
& \quad+\left\langle\left(\left(1-\beta_{n}\right) I-\alpha_{n} B\right)\left(T u_{n}-p\right), x_{n+1}-p\right\rangle \\
& \leq \quad \alpha_{n} \sigma\left\langle f\left(x_{n}\right)-f(p), x_{n+1}-p\right\rangle+\alpha_{n}\left\langle\sigma f(p)-B p, x_{n+1}-p\right\rangle \\
& \quad+\beta_{n}\left\|x_{n}-p\right\|\left\|x_{n+1}-p\right\|+\left(1-\beta_{n}-\alpha_{n} \gamma\right)\left\|T u_{n}-p\right\|\left\|x_{n+1}-p\right\| \\
& \leq {\left[1-(\gamma-\sigma \rho) \alpha_{n}\right]\left\|x_{n}-p\right\|\left\|x_{n+1}-p\right\|+\alpha_{n}\left\langle\sigma f(p)-B p, x_{n+1}-p\right\rangle } \\
& \leq \frac{1-(\gamma-\sigma \rho) \alpha_{n}}{2}\left\|x_{n}-p\right\|^{2}+\frac{1}{2}\left\|x_{n+1}-p\right\|^{2}+\alpha_{n}\left\langle\sigma f(p)-B p, x_{n+1}-p\right\rangle .
\end{aligned}
$$


It follows that

$$
\left\|x_{n+1}-p\right\|^{2} \leq\left[1-(\gamma-\sigma \rho) \alpha_{n}\right]\left\|x_{n}-p\right\|^{2}+2 \alpha_{n}\left\langle\sigma f(p)-B p, x_{n+1}-p\right\rangle .
$$

Applying Lemma 2.9 and (3.32) to (3.34), we deduce $x_{n} \rightarrow p$. The proof is completed.

Algorithm 3.3 Taking $x_{0} \in H_{1}$ arbitrarily, we define a sequence $\left\{x_{n}\right\}$ by the following:

$$
x_{n+1}=\alpha_{n} \sigma f\left(x_{n}\right)+\beta_{n} x_{n}+\left(\left(1-\beta_{n}\right) I-\alpha_{n} B\right) T\left(x_{n}+\delta A^{*}(S-I) A x_{n}\right)
$$

for all $n \in \mathbb{N}$, where $\delta \in\left(0, \frac{1}{\|A\|^{2}}\right)$ and $\sigma>0$ are two constants and $\left\{\alpha_{n}\right\}$ and $\left\{\beta_{n}\right\}$ are two real number sequences in $(0,1)$.

Corollary 3.4 Suppose $\Gamma_{1}=\{x \in \operatorname{Fix}(T): A x \in \operatorname{Fix}(S)\} \neq \emptyset$ and suppose the following conditions hold:

(C1): $\lim _{n \rightarrow \infty} \alpha_{n}=0$ and $\sum_{n=1}^{\infty} \alpha_{n}=\infty$;

(C2): $0<\liminf _{n \rightarrow \infty} \beta_{n} \leq \limsup _{n \rightarrow \infty} \beta_{n}<1$;

(C3): $\sigma \rho<\gamma$.

Then the sequence $\left\{x_{n}\right\}$ generated by algorithm (3.35) converges strongly to $p=\operatorname{Proj}_{\Gamma_{1}}(\sigma f+$ $I-B) p$, which solves the following VI:

$$
\langle(\sigma f-B) x, y-x\rangle \leq 0, \quad \forall y \in \Gamma_{1} .
$$

Algorithm 3.5 Taking $x_{0} \in H_{1}$ arbitrarily, we define a sequence $\left\{x_{n}\right\}$ by the following:

$$
x_{n+1}=\alpha_{n} \sigma f\left(x_{n}\right)+\beta_{n} x_{n}+\left(\left(1-\beta_{n}\right) I-\alpha_{n} B\right) U_{\lambda_{n}}^{F}\left(x_{n}+\delta A^{*}\left(U_{\gamma_{n}}^{G}-I\right) A x_{n}\right)
$$

for all $n \in \mathbb{N}$, where $\left\{\lambda_{n}\right\}$ and $\left\{\gamma_{n}\right\}$ are two real number sequences in $(0, \infty), \delta \in\left(0, \frac{1}{\|A\|^{2}}\right)$ and $\sigma>0$ are two constants and $\left\{\alpha_{n}\right\}$ and $\left\{\beta_{n}\right\}$ are two real number sequences in $(0,1)$.

Corollary 3.6 Suppose $\Gamma_{2}=\{x \in \operatorname{EP}(F): A x \in \operatorname{EP}(G)\} \neq \emptyset$ and suppose the following conditions hold:

(C1): $\lim _{n \rightarrow \infty} \alpha_{n}=0$ and $\sum_{n=1}^{\infty} \alpha_{n}=\infty$;

(C2): $0<\liminf _{n \rightarrow \infty} \beta_{n} \leq \limsup _{n \rightarrow \infty} \beta_{n}<1$;

(C3): $\liminf _{n \rightarrow \infty} \lambda_{n}>0$ and $\lim _{n \rightarrow \infty} \frac{\lambda_{n+1}}{\lambda_{n}}=1$;

(C4): $\liminf _{n \rightarrow \infty} \gamma_{n}>0$ and $\lim _{n \rightarrow \infty} \frac{\gamma_{n+1}}{\gamma_{n}}=1$;

(C5): $\sigma \rho<\gamma$.

Then the sequence $\left\{x_{n}\right\}$ generated by algorithm (3.36) converges strongly to $p=\operatorname{Proj}_{\Gamma_{2}}(\sigma f+$ $I-B) p$, which solves the following VI:

$$
\langle(\sigma f-B) x, y-x\rangle \leq 0, \quad \forall y \in \Gamma_{2} .
$$

Algorithm 3.7 Taking $x_{0} \in H_{1}$ arbitrarily, we define a sequence $\left\{x_{n}\right\}$ by the following:

$$
x_{n+1}=\alpha_{n} \sigma f\left(x_{n}\right)+\beta_{n} x_{n}+\left(\left(1-\beta_{n}\right) I-\alpha_{n} B\right) P_{C}\left(x_{n}+\delta A^{*}\left(P_{Q}-I\right) A x_{n}\right)
$$

for all $n \in \mathbb{N}$, where $\delta \in\left(0, \frac{1}{\|A\|^{2}}\right)$ and $\sigma>0$ are two constants and $\left\{\alpha_{n}\right\}$ and $\left\{\beta_{n}\right\}$ are two real number sequences in $(0,1)$. 
Corollary 3.8 Suppose $\Gamma_{3}=\{x \in C: A x \in Q\} \neq \emptyset$ and suppose the following conditions hold:

(C1): $\lim _{n \rightarrow \infty} \alpha_{n}=0$ and $\sum_{n=1}^{\infty} \alpha_{n}=\infty$;

(C2): $0<\liminf _{n \rightarrow \infty} \beta_{n} \leq \limsup _{n \rightarrow \infty} \beta_{n}<1$;

(C3): $\sigma \rho<\gamma$.

Then the sequence $\left\{x_{n}\right\}$ generated by algorithm (3.37) converges strongly to $p=\operatorname{Proj}_{\Gamma_{3}}(\sigma f+$ $I-B) p$, which solves the following VI:

$$
\langle(\sigma f-B) x, y-x\rangle \leq 0, \quad \forall y \in \Gamma_{3} .
$$

\section{Competing interests}

The authors declare that they have no competing interests.

\section{Authors' contributions}

All authors contributed equally and significantly in writing this paper. All authors read and approved the final manuscript.

\section{Author details}

${ }^{1}$ School of Mathematics and Information Science, Beifang University of Nationalities, Yinchuan, 750021, China. ${ }^{2}$ School of Mathematics and Information Technology, Nanjing Xiaozhuang University, Nanjing, 211171, China. ${ }^{3}$ Department of Information Management, Cheng Shiu University, Kaohsiung, 833, Taiwan. ${ }^{4}$ Center for Fundamental Science, Kaohsiung Medical University, Kaohsiung, 807, Taiwan. ${ }^{5}$ Department of Mathematics, Tianjin Polytechnic University, Tianjin, 300387, China

\section{Acknowledgements}

Li-Jun Zhu was supported in part by NSFC 61362033 and NZ13087. Yeong-Cheng Liou was supported in part by NSC 101-2628-E-230-001-MY3 and NSC 101-2622-E-230-005-CC3.

\section{Received: 29 March 2014 Accepted: 11 September 2014 Published: 02 Oct 2014}

\section{References}

1. Censor, Y, Elfving, T: A multiprojection algorithm using Bregman projections in a product space. Numer. Algorithms 8 221-239 (1994)

2. Byrne, C: Iterative oblique projection onto convex subsets and the split feasibility problem. Inverse Probl. 18, 441-453 (2002)

3. Ceng, LC, Ansari, QH, Yao, JC: An extragradient method for split feasibility and fixed point problems. Comput. Math. Appl. 64, 633-642 (2012)

4. Dang, Y, Gao, Y: The strong convergence of a KM-CQ-like algorithm for a split feasibility problem. Inverse Probl. 27 $015007(2011)$

5. Wang, F, Xu, HK: Approximating curve and strong convergence of the CQ algorithm for the split feasibility problem. J. Inequal. Appl. 2010, Article ID 102085 (2010). doi:10.1155/2010/102085

6. Xu, HK: Iterative methods for the split feasibility problem in infinite-dimensional Hilbert spaces. Inverse Probl. 26, $105018(2010)$

7. Yang, Q: The relaxed CQ algorithm solving the split feasibility problem. Inverse Probl. 20, 1261-1266 (2004)

8. Yao, Y, Wu, J, Liou, YC: Regularized methods for the split feasibility problem. Abstr. Appl. Anal. 2012, Article ID 140679 (2012). doi:10.1155/2012/140679

9. Yao, Y, Kim, TH, Chebbi, S, Xu, HK: A modified extragradient method for the split feasibility and fixed point problems. J. Nonlinear Convex Anal. 13, 383-396 (2012)

10. Zhao, J, Yang, Q: Several solution methods for the split feasibility problem. Inverse Probl. 21, 1791-1799 (2005)

11. Qu, B, Xiu, N: A note on the CQ algorithm for the split feasibility problem. Inverse Probl. 21, 1655-1665 (2005)

12. Wang, Z, Yang, Q, Yang, Y: The relaxed inexact projection methods for the split feasibility problem. Appl. Math. Comput. (2010). doi:10.1016/j.amc.2010.11.058

13. Ceng, LC, Ansari, QH, Yao, JC: Relaxed extragradient methods for finding minimum-norm solutions of the split feasibility problem. Nonlinear Anal. 75, 2116-2125 (2012)

14. Censor, Y, Segal, A: The split common fixed point problem for directed operators. J. Convex Anal. 16, 587-600 (2009)

15. Moudafi, A: The split common fixed-point problem for demi-contractive mappings. Inverse Probl. 26, 587-600 (2010)

16. Wang, F, Xu, HK: Cyclic algorithms for split feasibility problems in Hilbert spaces. Nonlinear Anal. 74, 4105-4111 (2011)

17. Moudafi, A: A note on the split common fixed-point problem for quasi-nonexpansive operators. Nonlinear Anal. 74 , 4083-4087 (2011)

18. He, ZH: The split equilibrium problems and its convergence algorithms. J. Inequal. Appl. 2012, 162 (2012)

19. He, ZH, Du, WS: Nonlinear algorithms approach to split common solution problems. Fixed Point Theory Appl. 2012, $130(2012$

20. Moudafi, A: Split monotone variational inclusions. J. Optim. Theory Appl. 150, 275-283 (2011)

21. Byrne, C, Censor, Y, Gibali, A, Reich, S: The split common null point problem. J. Nonlinear Convex Anal. 13, 759-775 (2012) 
22. Censor, Y, Gibali, A, Reich, S: Algorithms for the split variational inequality problem. Numer. Algorithms 59, $301-323$ (2012)

23. Yao, Y, Marino, G, Muglia, L: A modified Korpelevich's method convergent to the minimum norm solution of a variational inequality. Optimization 63, 559-569 (2014)

24. Yao, Y, Marino, G, Xu, HK, Liou, YC: Construction of minimum norm fixed points of pseudocontractions in Hilbert spaces. J. Inequal. Appl. 2014, 206 (2014)

25. Chang, SS, Wang, L, Tang, YK, Yang, L: The split common fixed point problem for total asymptotically strictly pseudocontractive mappings. J. Appl. Math. 2012, Article ID 385638 (2012)

26. Blum, E, Oettli, W: From optimization and variational inequalities to equilibrium problems. Math. Stud. 63, 123-145 (1994)

27. Moudafi, A: Weak convergence theorems for nonexpansive mappings and equilibrium problems. J. Nonlinear Convex Anal. 9, 37-43 (2008)

28. Yao, Y, Noor, MA, Liou, YC: On iterative methods for equilibrium problems. Nonlinear Anal. 70, 497-507 (2009)

29. Ceng, LC, Al-Homidan, S, Ansari, QH, Yao, JC: An iterative scheme for equilibrium problems and fixed point problems of strict pseudocontraction mappings. J. Comput. Appl. Math. 223, 967-974 (2009)

30. He, ZH, Du, WS: On hybrid split problem and its nonlinear algorithms. Fixed Point Theory Appl. 2013, $47(2013)$

31. Combettes, PL, Hirstoaga, A: Equilibrium programming in Hilbert spaces. J. Nonlinear Convex Anal. 6, 117-136 (2005)

32. Cianciaruso, F, Marino, G, Muglia, L, Yao, Y: A hybrid projection algorithm for finding solutions of mixed equilibrium problem and variational inequality problem. Fixed Point Theory Appl. 2010, Article ID 383740 (2010)

33. Suzuki, T: Strong convergence theorems for infinite families of nonexpansive mappings in general Banach spaces. Fixed Point Theory Appl. 2005, 103-123 (2005)

34. Geobel, K, Kirk, WA: Topics in Metric Fixed Point Theory. Cambridge Studies in Advanced Mathematics, vol. 28 Cambridge University Press, Cambridge (1990)

35. Xu, HK: Iterative algorithms for nonlinear operators. J. Lond. Math. Soc. 66, 240-256 (2002)

10.1186/1029-242X-2014-380

Cite this article as: Zhu et al.: Superimposed algorithms for the split equilibrium problems and fixed point problems. Journal of Inequalities and Applications 2014, 2014:380

\section{Submit your manuscript to a SpringerOpen ${ }^{\circ}$ journal and benefit from:}

- Convenient online submission

Rigorous peer review

- Immediate publication on acceptance

- Open access: articles freely available online

- High visibility within the field

- Retaining the copyright to your article 